\title{
A Structure-based Proposal for the Catalytic Mechanism of the Bacterial Acid Phosphatase AphA belonging to the DDDD Superfamily of Phosphohydrolases
}

\author{
Vito Calderone ${ }^{1}$, Costantino Forleo ${ }^{1,2}$, Manuela Benvenuti ${ }^{1}$ \\ Maria Cristina Thaller $^{3}$, Gian Maria Rossolini ${ }^{2}$ and Stefano Mangani ${ }^{1 *}$
}

${ }^{1}$ Dipartimento di Chimica Università di Siena, Via Aldo Moro, I-53100 Siena, Italy

${ }^{2}$ Dipartimento di Biologia Molecolare, Università di Siena I-53100 Siena, Italy

${ }^{3}$ Dipartimento di Biologia Università di Roma "Tor Vergata", I-00133 Rome, Italy

${ }^{*}$ Corresponding author

\begin{abstract}
The Escherichia coli gene aphA codes for a periplasmic acid phosphatase called AphA, belonging to class B bacterial phosphatases, which is part of the DDDD superfamily of phosphohydrolases. After our first report about its crystal structure, we have started a series of crystallographic studies aimed at understanding of the catalytic mechanism of the enzyme. Here, we report three crystal structures of the AphA enzyme in complex with the hydrolysis products of nucleoside monophosphate substrates and a fourth with a proposed intermediate analogue that appears to be covalently bound to the enzyme. Comparison with the native enzyme structure and with the available X-ray structures of different phosphatases provides clues about the enzyme chemistry and allows us to propose a catalytic mechanism for AphA, and to discuss it with respect to the mechanism of other bacterial and human phosphatases.
\end{abstract}

(C) 2005 Elsevier Ltd. All rights reserved.

Keywords: class B phosphatase; bacterial phosphatase; DDDD phosphohydrolase superfamily; crystal structure; catalytic mechanism

\section{Introduction}

Bacterial class B acid phosphatases (CBAPs) are a group of secreted enzymes that have been detected in a minority of bacterial species, but these include a number of major pathogens such as members of the family Enterobacteriaceae (e.g. Escherichia coli, Salmonella enterica, Shigella flexneri, Klebsiella pneumoniae and Proteus mirabilis), Haemophilus

Abbreviations used: ADN, adenosine; AphA, aspecific acid phosphatase; $\beta$-PGM, $\beta$-phosphoglucomutase; CBAPs, class $B$ acid phosphatases; CMP, cytidine $5^{\prime}$-monophosphate; dCMP, $2^{\prime}$-deoxyctidine-

5'-monophosphate; dCTN, 2'-deoxycitidine; dNT-2, human mitochondrial $5^{\prime}\left(3^{\prime}\right)$-deoxyribonucleotidase;

HAD, haloacid dehalogenase; 8-KDO, deoxy-D-mannoseoctulosonate 8-phosphate phosphatase; MDP-1, magnesium dependent phosphatase 1; PLS, phospho-l-serine; PNPP, para-nitrophenylphosphate; PSP, phosphoserine phosphatase; RMSD, root-mean-square deviation; T6P, trehalose-6-phosphate phosphatase; TM0651,

phosphatase TM0651 from Thermotoga maritima.

E-mail address of the corresponding author: mangani@unisi.it influenzae, Actinobacillus actinomycetemcomitans, Streptococcus pyogenes and Streptococcus agalactiae.

CBAPs belong to the DDDD superfamily of phosphohydrolases, a large protein lineage with representatives in the three living kingdoms that, although highly diverse at the level of primary structure and function, share some conserved sequence motifs, including four highly conserved aspartate residues. ${ }^{1}$

The E. coli AphA enzyme is the only CBAP whose three-dimensional structure has been determined. ${ }^{2}$ The enzyme is made of four identical $25 \mathrm{kDa}$ subunits assembled to form a flat molecule with two catalytic sites on each face. Each catalytic site contains a $\mathrm{Mg}$ (II) ion that can be replaced by other metal ions, and that is coordinated by three of the four conserved aspartate residues typical of the DDDD superfamily; namely, Asp44, Asp46 and Asp167. The AphA subunit exhibits a haloacid dehalogenase-like (HAD) fold ${ }^{2}$ similar to that of other Mg-phosphatases belonging to the DDDD superfamily, such as the phosphoserine phosphatases of Methanococcus jannaschii ${ }^{3,4}$ and Homo sapiens, ${ }^{5}$ the enzyme TM0651 from Thermotoga maritima ${ }^{6}$ or the Thermoplasma acidophilum phospho- 
glycolate phosphatase. ${ }^{7}$ Despite the apparent lack of sequence homology, this fold is shared by other Mg-dependent phosphatases not included in the DDDD superfamily, such as the human mitochondrial $5^{\prime}\left(3^{\prime}\right)$-deoxyribonucleotidase $(\mathrm{dNT}-2){ }^{8}$ Mechanistic proposals based on crystallographic evidence have been made recently about phosphoserine phosphatase $(p s p)^{3-5}$ and $\mathrm{dNT}-2{ }^{8}$ and the catalytic mechanism of the phosphatases/phosphotransferases of the HAD superfamily has been described and rationalized very recently. ${ }^{9}$

The HAD phosphatases are characterized by the occurrence of three conserved sequence motifs: motif 1 consists of DXDX[T/V][L/V $],{ }^{10}$ where the first Asp residue is the nucleophile attacking the substrate phosphate group, ${ }^{3}$ motif 2 is [S/T], where a Ser/Thr residue binds the substrate phosphoryl group by $\mathrm{H}$-bonding; finally, motif 3 is $\mathrm{K}-(\mathrm{X})_{n}-[\mathrm{G} / \mathrm{S}]$ $[\mathrm{D} / \mathrm{S}](\mathrm{X})_{n}[\mathrm{D} / \mathrm{N}]{ }^{3,11-13}$ which is part of the active site and is involved in orienting the Asp nucleophile, the substrate phosphate group and in binding the catalytic $\mathrm{Mg}$ (II) ion. ${ }^{14}$ The HAD phosphatases are further classed into three subfamilies (I-III), depending on the occurrence of and on the extent of a domain that functions as a "cap" over the active site domain. ${ }^{9,15}$ Subfamily I is characterized by a relatively small cap domain inserted between motifs 1 and 2 (e.g. PSP), ${ }^{3}$ subfamily II has a large cap domain inserted between motifs 2 and 3 (e.g. TM0651), ${ }^{6}$ while subfamily III is characterized by the absence of the cap domain (e.g. MDP-1; 8-KDO). ${ }^{15-17}$

AphA exhibits optimal activity at slightly acidic $\mathrm{pH}$, and it is active on several substrates including p-nitrophenyl phosphate, $5^{\prime}$ and $3^{\prime}$-mononucleotides, $\beta$-glycerol phosphate, and sugar-phosphates. ${ }^{18}$ However, it exhibits a strong preference for $5^{\prime}$ and $3^{\prime}$-mononucleotide substrates $\left(k_{\text {cat }} / K_{\mathrm{M}}\right.$ usually $>10^{6} \mathrm{M}^{-1} \mathrm{~s}^{-1}$ ) and for other organic phosphomonoesters with an aromatic ring moiety. AphA can also catalyse the transfer of phosphate from phosphomonoester donors (e.g. p-nitrophenyl phosphate, $3^{\prime}$ or $5^{\prime}$-mononucleotides) to the hydroxyl groups of other organic compounds (e.g. nucleosides) acting as acceptors. ${ }^{18}$

The comparison of the AphA structure ${ }^{2}$ with that of the $\mathrm{Mg}$ phosphatases of the HAD fold, shows similarities suggesting that AphA should share overall mechanistic features with these enzymes, while the substrate specificity and the details of the catalytic mechanism would be determined by the unique features presented by AphA.

Here, we describe the crystallographic analysis of AphA complexes with inhibitors and reaction products that allowed us to propose a catalytic mechanism for AphA on the basis of analogies with other Mg-dependent phosphatases/phosphotransferases of the HAD superfamily.

\section{Results}

We have determined the crystal structures of AphA complexes with the reaction products of the substrates AMP (two structures, PDB codes 2B8J and 2B82) and dCMP (PDB code 1RMY) and with osmate (PDB code 1RMQ). Unlike the native I222 crystal, $^{2}$ all the AphA complexes belong to the monoclinic space group $C 2$. In any case, the quaternary structure of AphA consists of a homotetramer with point symmetry $222,{ }^{2}$ as shown in Figure 1. Each face of the molecule contains two catalytic sites where the essential magnesium ions are bound. The active site cleft consists of a long and narrow channel (about $25 \AA \times 10 \AA$ ) whose walls are made by helix 2 and loop 6 on one side, and by helix 7 and loop 7 on the other. The $\mathrm{Mg}$ (II) ion, coordinated by the carboxylate groups of Asp44 and Asp167 and the backbone carbonyl oxygen atom of Asp46, lies at the bottom of the cleft about $10 \AA$ from the protein surface. The active site cavity is characterized by the presence of three further aspartate residues (conserved in class B phosphatases), Asp45, Asp169 and Asp171. The only positively charged residues present in the active site, which are conserved also in class B phosphatases, are Arg114, Arg187 and Lys152, the latter being located at the beginning of helix 6 , just at the end of loop 6 and conserved in class B and C phosphatases. Table 1 reports the sequence alignment of class B phosphatases. ${ }^{19}$

Table 2 reports a summary of the asymmetric unit content in each crystal, the type of ligands bound to the enzyme and the conformation of loop 6 .
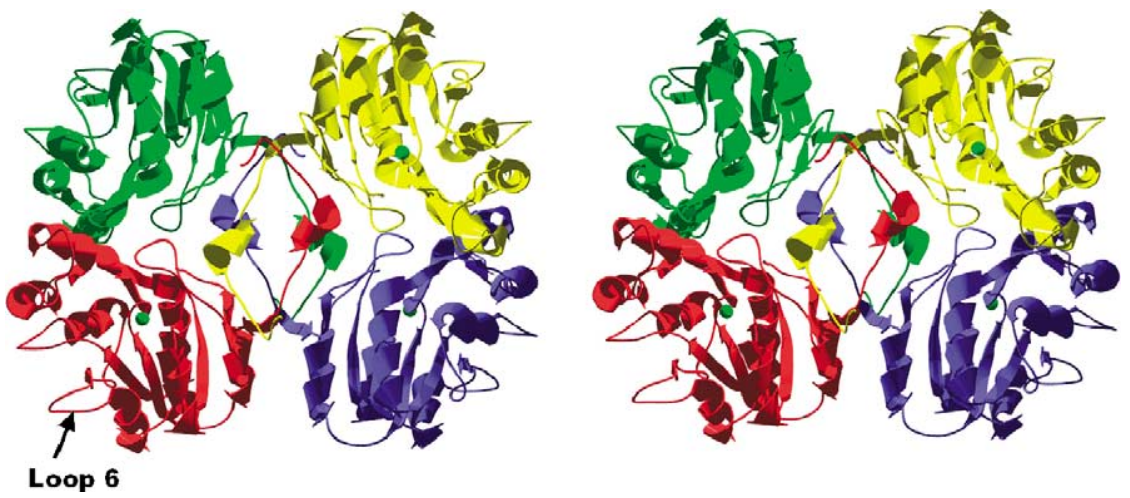

Figure 1. Quaternary structure of AphA. Each subunit is represented in different colors. The $\mathrm{Mg}$ (II) ions are represented as green spheres of arbitrary radius. The mobile loop 6 is labeled and indicated by an arrow. 
Table 1. Sequence alignment of class B phosphatases on E. coli AphA

\section{$44 \quad 46$}

Apha-ECO

AphA-Shflex

AphA-Salti

AphA-Kpn

AphA-Mmo

AphA-Actact

AphA-Hin

AphA-SagaI

AphA - Spyo

Apha $-E C O$

AphA-Shflex

AphA-Salti

AphA-Kpn

AphA-Mmo

AphA-Actact

AphA-Hin

AphA-Sagal

AphA-Spyo
Apha-ECO

AphA-Shflex

AphA-Salti

AphA-Kpn

AphA-Mmo

AphA-Actact

AphA-Hin

AphA-Sagal

AphA-Spyo

Apha-ECO

AphA-Shflex

AphA-Salti

AphA-Kpn

AphA-Mmo

AphA-Actact

AphA-Hin

AphA-Sagal

AphA-Spyo

- - SSPSPLNPGTNVARLAEQAP IHWVSVAQIENSLAGRPPMAVGFDIDDTVLFSSPGFWR - - SSPSPLNPGTNVAKLAEQAP IHWVSVAQIENSLAGRPPMAVGFDIDDTVLFSSPGFWR - -SSPSTLNPGTNVAKLAEQAPVHWVSVAQIENSLTGRPPMAVGFDIDDTVLFSSPGFWR - - STPQPLWVGTNVAQLAEQAP IHWVSVAQIENSLLGRPPIAVGFDIDDTVLFSSPGFSR KVYMPEKVSDGVTVAQLAEQHAIHWISVEQIEESLKGQP - MAVGFDIDDTVLFSSPGFYR - AKSEPYTHQGTNAREMLVEQPIHWISVDQLKKELEGKAPMNVSFDIDDTVLFSSPCFYH - GKTEPYTQSGTNAREMLQEQAIHWISVDQI KQSLEGKAP INVSFDIDDTVLFSSPCFYH KGPKVAYTQEGMTALSDTNKDKVTTISIDEIQKSLEGKKPITVSFDIDDTLLFSSQYFQY SGPKVPYTQEGITAIS - NQATVKLISIADIASSLEGQKPITVSFDIDDTLLFTSQYFQY

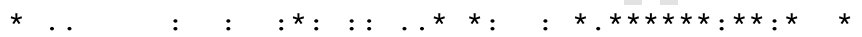

114

GKKTFSPESEDYLKNPVFWEKMNNGWDEFS I PKEVARQLIDMHVRRGDAIFFVTGRS - - GKKTFSPESEDYLKNPVFWEKMNNGWDEFS I PKEVARQLIDMHVRRGDAIFFVTGRS - - GKKTYSPDSDDYLKNPAFWEKMNNGWDEFS I PKEVARQLIDMHVRRGDS IYFVTGRS - - GQKTFSPGSEDYLKNPQFWEKMNNGWDEFSMPKEVARHLIAMHVKRGDSIWFVTGRS - - GKLEYSPNDYSYLKNPEFWEKMNNEWDKFSMPKKSGMELVQMHLKRGDTVYFITGRS - - GQQKYSPGKQDYLKNQDFWNEVNAGCDQYS I PKQIAVDLINMHQERGDQIYFITGRT - - GQQKF SPGKHDYLKNQDFWNEVNAGCDKYS I PKQIAIDLINMHQARGDQVYFFTGRT - - GKEYVTPGSFDFLHKQKFWDLVAKRGDQDS I PKEYAKKLIAMHQKRGDKIVFITGRTRGS GKEYITPGSFDFLHKQKFWDLVAKRGDQDS I PKEYAKQLIAMHQKRGDKIVFITGRTRGS

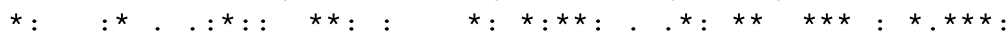

145 152 167

- - - PTKTET-VSKTLADNFHIPATNMNPVIFAG - - D- - -KPGQNTKSQWLQDKNIRIFYGD - PTKTET-VSKTLADNFHI PATNMNPVIFAG - - D- - KPGQNTKSQWLQDKNIRIFYGD - - -QTKTET-VSKTLADNFHI PAANMNPVIFAG - - - - KPGQNTKVQWLQEKNMRIFYGD - - QTKTET-VSKTLQDDFLIPAANMNPVIFAG - - D- - KPGQNTKTQWLQAKQI KVFYGD - - - KTKTET-VTKYVQEGLRI PADKMNPVIFAG - - D - - EEGQNNKVSWMRDHKLKIYYGD - - AGDKDG-VPPVLQKAFSIK- -NMHPVEFMGGRD- - RTTKYNKTPGIIEHKVTIHYGD - - AGKVDG-VTPILEKTFNIK- -NMHPVEFMGSRE - - RTTKYNKTPAI I SHKVS IHYGD MYKEGEVDK-TAKALAKDFKLD- - KPIAVNYTG- - DKPKKPYKYDKSYYIKKYGSDIHYGD MYKKGEIDK-TAKSLAKDFKLD- -KPIAINYTG- -DKAVKPYQYDKTYYIKKNGSQIHYGD

171

169

187

SDNDITAARDVGARGIRILRASNSTYKPLPQAGAFGEEVIVNSEY SDNDITAARDVGARGIRILRASNSTYKPLPQAGAFGEEVIVNSEY SDNDITAARDCGIRGIRILRAANSTYKPLPQAGAFGEEVIVNSEY SDNDITAAREAGARGIRVLRAANSSYKPLPMAGALGEEVIVNSEY ADADIAAARELNIRGIRVLRASNSSYQPLPKAGQFGEEVVINSEY SDDDILAAKEAGVRGIRLMRAANSTYQPMPTLGGYGEEVLINSSY SDDDVLAAKEAGVRGIRLMRAANSTYQPMPTLGGYGEEVLINSSY SDDDIHAAREAGARP IRILRAPNSTNLPLPEAGGYGEEVLENSAY SDEDINAAKEAGARP IRILRAPNSTNLPLPKAGGYGEEVLENSAY

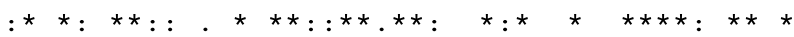

Apha-Eco, Escherichia coli AphA, P32697; AphA-Shflex, Shigella flexneri AphA; AphA-Salti, Salmonella enterica serovar typhi AphA, O08430; AphA-Kpn, Klebsiella pneumoniae AphA, Q8VQS4; AphA-Mmo, Morganella morganii, Q59544; AphA-Actact, Actinobacillus actinomycetemcomitans unfinished genome-Actinobacillus Genome Sequencing Project, at the Oklahoma University; AphA-Hin, Haemophilus influenzae AphA, P44009; AphA-Sagal, Streptococcus agalactiae AphA, Q8E0T5; AphA-Spyo, Streptococcus pyogenes AphA, Q7CN89. The asterisk $\left(^{*}\right)$ denotes strictly conserved residues, the column denotes conserved similar residues and the dot denotes partly conserved residues. The highlighted residues are $\mathrm{Mg}$ (II) ligands; those in bold are located in the active site. In some Escherichia and Shigella genome sequences, aphA gene products have been annotated erroneously as diadenosine tetraphosphatase (apaH), most probably due to the similarity of names; class B phosphatases, however, share no similarity with ApaH enzymes. P44009 includes an M73 residue, that has been not considered in this alignment, as it is absent from all the other available deduced sequences of this protein and possibly arises from the amending of an artificial frame-shift in U32731. (Sequences alignment: NPS at Network Protein Sequence Analysis.) ${ }^{19}$

\section{AphA complexed with AMP reaction products at high resolution}

The structure of the native AphA crystals soaked with the substrate AMP has been determined at $1.25 \AA$ resolution (2B82). The structure shows a ternary complex between AphA and the hydrolysis products of AMP, adenosine and phosphate, which are bound at different locations within the active site crevice. The ternary complex crystallises in the C2 space group with the AphA molecule sitting on a 2 -fold axis in the crystal, hence the asymmetric unit contains two crystallographically independent AphA subunits (Table 2). The two subunits have 
Table 2. Asymmetric unit content, ligands bound and conformation of loop 6 in each AphA crystal structure

\begin{tabular}{|c|c|c|c|c|}
\hline Compound & AphA-ADN-P $1.25 \AA$ & AphA-osmate & AphA-ADN-P $2.0 \AA$ & AphA-dCTN-P \\
\hline PDB code & 2B82 & 1RMQ & 2B8J & 1RMY \\
\hline Space group & $\mathrm{C} 2$ & $\mathrm{C} 2$ & $\mathrm{C} 2$ & $\mathrm{C} 2$ \\
\hline $\begin{array}{l}\text { Asymmetric unit } \\
\text { content }\end{array}$ & 2 subunits ( $\mathrm{A}$ and $\mathrm{B})$ & 2 subunits $(\mathrm{A}$ and $\mathrm{B})$ & 2 subunits (A and $B)$ & 2 subunits (A and $B)$ \\
\hline Ligands & $\begin{array}{l}\text { Adenosine }+ \text { phosphate } \\
\text { in both subunits } \\
\text { A and B }\end{array}$ & $\begin{array}{l}\text { Tetrahedral osmate } \\
\text { species in A; five } \\
\text { coordinate osmate } \\
\text { species in B }\end{array}$ & $\begin{array}{l}\text { Au(spermine) } \\
\text { complex }+ \text { phosphate } \\
\text { in A; adenosine alone } \\
\text { in B }\end{array}$ & $\begin{array}{l}\text { Deoxycitidine alone } \\
\text { in A; deoxycitidine }+ \\
\text { phosphate in B }\end{array}$ \\
\hline Loop 6 conformation & Closed in both subunits & Closed in $\mathrm{A}$; open in $\mathrm{B}$ & Closed in $\mathrm{A}$; open in $\mathrm{B}$ & Open in $\mathrm{A}$; closed in $\mathrm{B}$ \\
\hline
\end{tabular}

identical structure (RMSD on backbone atoms $0.30 \AA$ ) with the only variation of a different orientation of the N-terminal Ser-Pro-Ser peptide. The phosphate anions have well-defined electron density allowing refinement at full occupancy in both crystal subunits, and the structure indicates identical binding modes. In contrast, the electron density for the adenosine molecule is less clear. The shape of the electron density corresponding to the aromatic moiety of the molecule suggests the presence of rotational disorder over several positions obtained by rotation around the purine ring. Two main orientations of the nucleoside have been modelled and refined at $50 \%$ occupancy. The final model of the AphA-nucleoside-phosphate complex is reported in Figure 2(a) and (b) superimposed on the electron density map. The hydrolysed phosphate establishes a coordination bond with the catalytic $\mathrm{Mg}$ (II) ion by replacing one of the three water molecules present in the native $\mathrm{Mg}(\mathrm{II})$ coordination sphere. The other interactions of phosphate with the cavity consists of several H-bonds with the side-chains of Asp44, Asp46, Thr112, Lys152 and with the backbone NH of Ile45, Asp46 and Gly113 (Figure 3). Asp44, Asp46, Thr48 and Val49 constitute the motif 1 of the HAD superfamily. $^{10}$ Thr112 constitutes motif 2 of HAD, ${ }^{9,20}$ while Lys152, together with Gly166, Asp167 and Asp171, compose motif 3 of HAD phosphatases. ${ }^{3,11-13}$ The comparison of AphA with the HAD Mg-phosphatases, shows that AphA falls somewhere in between the subfamily I and III having a rigid "cap" domain, 9 much smaller than that of PSP and larger than that of 8-KDO.

The structure indicates that the phosphatebinding site is one of the reaction product-binding sites (referred to as the P1 site). Least-squares superposition of the backbone atoms of Asp44,
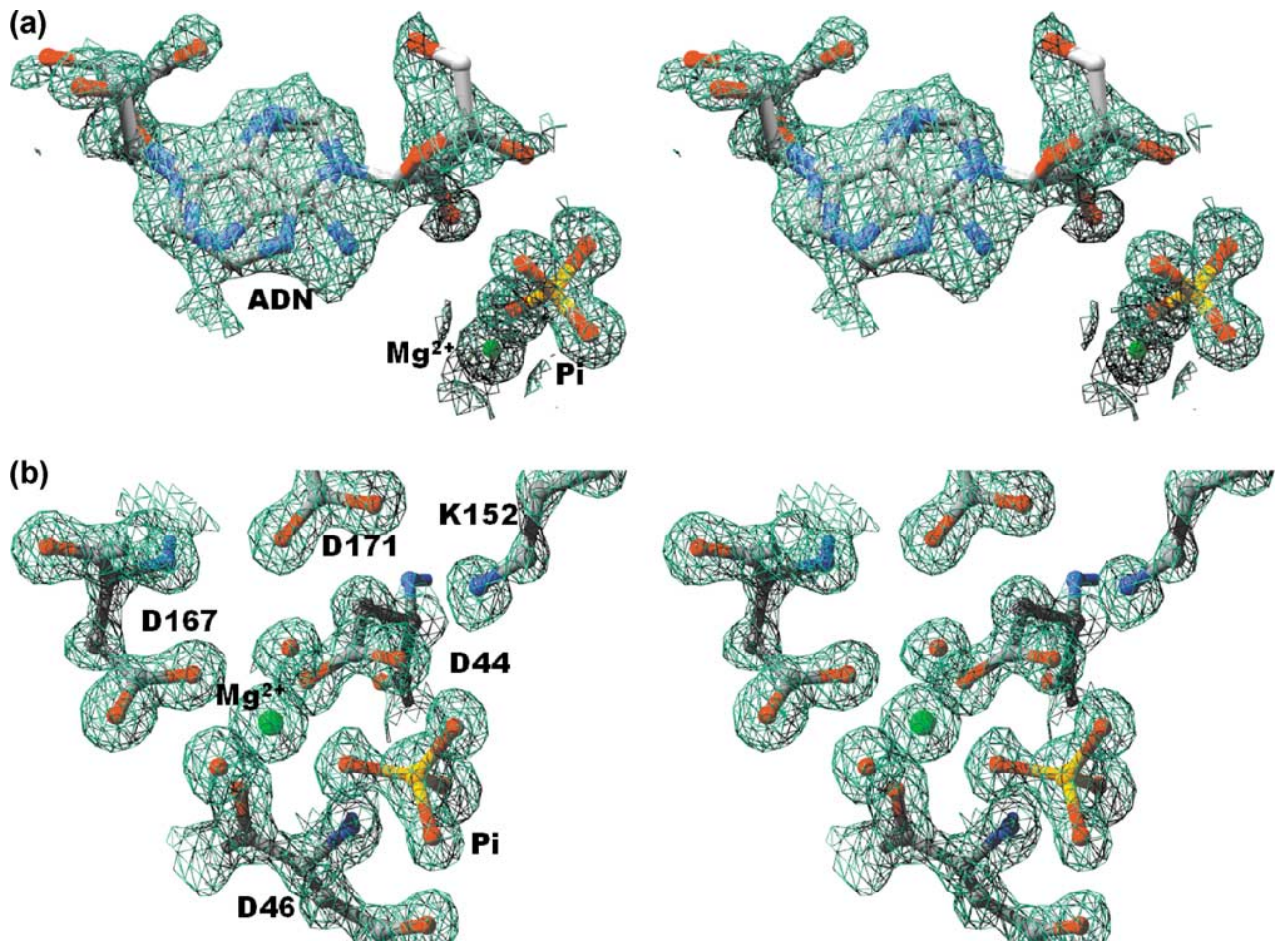

Figure 2. (a) Detail of the AphA-ADN- $P_{i}$ complex showing the $2 F_{\mathrm{o}}-F_{\mathrm{c}}$ electron density map (contoured at $1.0 \sigma$ and calculated with phases from the final refined model of the ternary complex at $1.25 \AA$ ), corresponding to the ADN molecule, the $\mathrm{Mg}(\mathrm{II})$ ion and $\mathrm{P}_{\mathrm{i}}$. The map is superimposed on the two main ADN refined orientations used to model the disorder present in the site. (b) The $\mathrm{Mg}$ (II) site portion of the above map superimposed on the AphA active site final model. Notice the orientation of the phosphate anion positioning the $\mathrm{P}$ atom directly in front of the Asp44 side-chain $\mathrm{O}$ atom. 

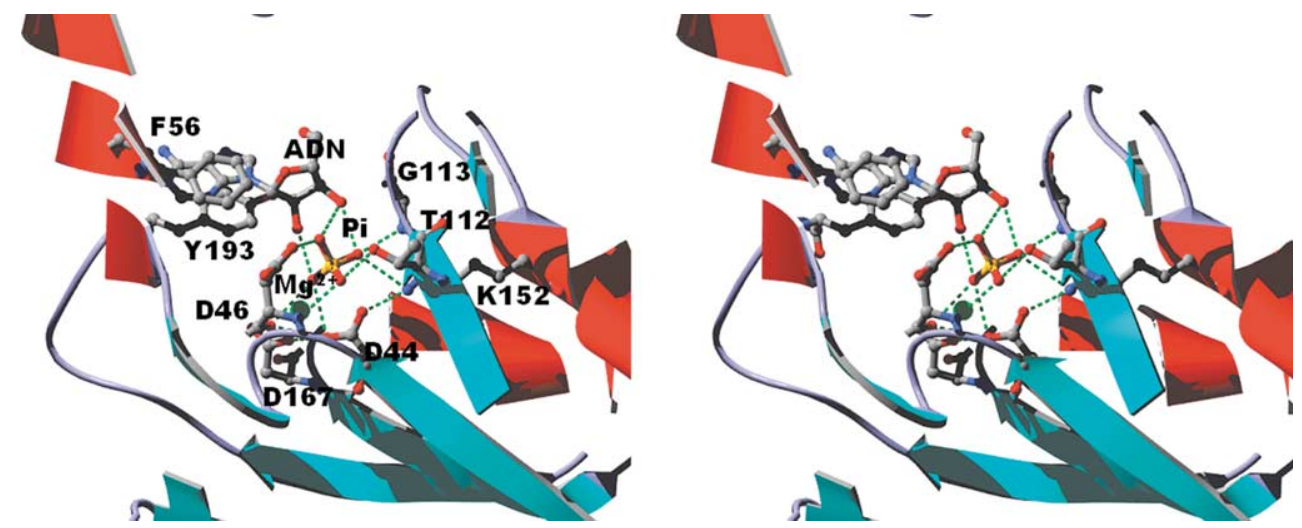

Figure 3. The H-bonding scheme involving the $\mathrm{P}_{\mathrm{i}}$ and $\mathrm{ADN}$ reaction products bound to the active site of AphA.

Ile45 and Asp46 of AphA-adenosine phosphate with the corresponding three residues of phosphate-inhibited (1L7M) and phospho-l-serine (PLS)-bound PSP (1L7P), Asp11, Phe12 and Asp $13^{5}$ shows a perfect correspondence of the phosphate positions in all the three structures. This finding suggests that, in analogy with the structure of the PLS-PSP complex, the phosphate position in the present ternary complex might also represent the docking mode of the phosphate moiety of intact AMP to AphA.

It is interesting to note that the Asp46 carboxylate oxygen atom is $\mathrm{H}$ bonded to phosphate in the anti conformation due to the salt-link that the carboxylate engages with the $\mathrm{N}^{\varepsilon}$ and $\mathrm{N}^{\eta}$ hydrogen atoms of the Arg114 residue, which is conserved in all class B phosphatases (Table 1). The phosphate is also $\mathrm{H}$ bonded to the $\mathrm{OH} 2$ and $\mathrm{OH} 3$ hydroxyl groups of the adenine ribose ring, and is situated on the opposite side with respect to the ribose $\mathrm{O} 5$ atom from which it has been cleaved (in one of the modelled conformations). The aromatic moiety of the adenosine ring fits in a hydrophobic narrow cleft delimited by the stacked side-chains of Phe56 and Tyr193, and by Tyr70 and Leu71 as shown in Figures 3 and 4 . The adenosine-binding site represents the binding site of the second hydrolysis product of the reaction (P2 site). The contact distance between the ADN ribose ring and the cleaved phosphate allows us to propose that the hydrophobic pocket between Phe56 and Tyr198 constitutes also the binding site of the substrate aromatic moiety, indeed, modelling of AMP purine ring into this pocket brings the phosphate group into coordination distance from the $\mathrm{Mg}$ (II) ion. $^{21}$ This cleft clearly accounts for the preference of AphA for aromatic substrates. ${ }^{2,18}$

Least-squares superposition of the subunits of the present ternary complex with the subunits of native (PDB code $1 \mathrm{~N} 9 \mathrm{~K}$ ) and $\mathrm{Au}^{3+}$-bound (PDB code $1 \mathrm{~N} 8 \mathrm{~N})$ AphA confirmed very similar overall fold with RMSDs on the backbone atoms between $0.30 \AA$ and $0.32 \AA$. However, the superposition shows that loop 6 (residues 143-151), linking the short strand 4 to helix $6,{ }^{2}$ adopts a quite different conformation in the present ternary complex with respect to both ligand-free forms of the enzyme (1N9K and 1N8N). As shown in Figure 4, this loop is open towards the solvent in the native enzyme, while it is bent over towards the active site in both subunits of the ternary complex.

The movement of loop 6 (residues 143-151) described above allows the establishment of different interactions of the side-chains of Asp145,
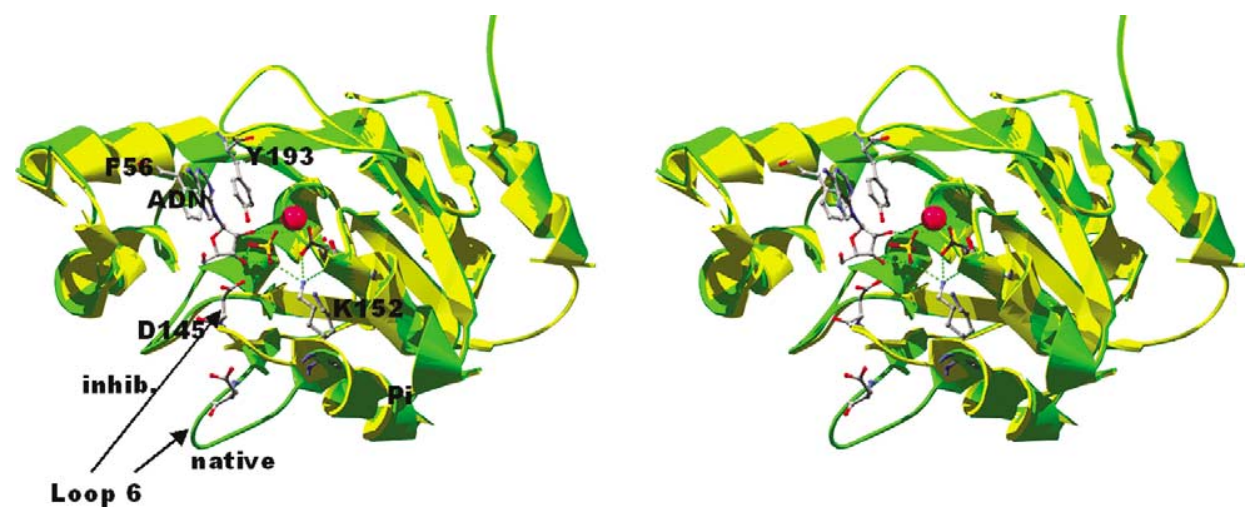

Figure 4. Detail of the least-squares superposition of native AphA (green) with the AphA-ADN- $\mathrm{P}_{\mathrm{i}}$ ternary complex (yellow) showing the loop 6 movement upon ligand binding. Relevant residues of the active site and the $P_{i}$ and ADN ligands bound to the P1 and P2 sites, respectively, are also shown as stick models. 


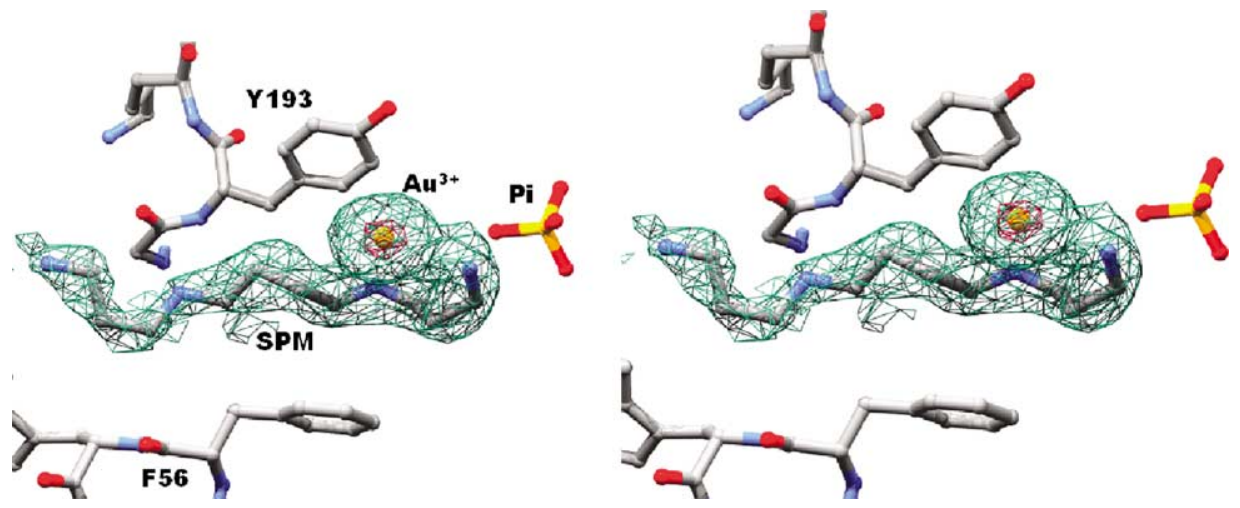

Figure 5. Detail of the $2 F_{\mathrm{o}}-F_{\mathrm{c}}$ electron density map reporting the $\mathrm{Au}(\mathrm{III})$-spermine adduct bound into the $\mathrm{P} 2$ ringbinding cleft of the AphA-ADN-P $P_{i}$ complex at $2.0 \AA$ resolution. The $2 F_{o}-F_{c}$ map (cyan) is contoured at $1.0 \sigma$, superimposed on an omit map (red) contoured at $10.0 \sigma$ showing the position of the $\mathrm{Au}(\mathrm{III})$ ion.

Asn150 and Lys152 with respect to the native enzyme. In the two subunits, the Lys152 residue is driven towards the active site by establishing strong hydrogen bonds with the carboxylate groups of Asp44 (2.6 $\AA$ ) and Asp171 (2.8 $\AA$ ), with the carbonyl oxygen atom of Val111 (2.9 $\AA$ ) and with one of the phosphate oxygen atoms $(2.8 \AA)$. In the native AphA (1N8N), Lys152 interacts only with Asp171, remaining far from the active site. Following the movement of loop 6, the Asn150 side-chain is within $\mathrm{H}$ bonding distance of the side-chain of Asp177, while Asp145 reaches the ribose ring of adenosine.

Significant extra electron density, which can be interpreted as a third adenosine molecule at partial occupancy, is found on the external wall of the helix2-loop3-helix3 motif of subunit $\mathrm{B}^{2}$ In this respect, is interesting to note that adenosine behaves as a non-competitive inhibitor of AphAcatalysed hydrolysis of PNPP (unpublished data).

\section{AphA complexed with AMP reaction products at 2.0 A resolution}

The structure of a second AphA ternary complex with adenosine and phosphate has been obtained at $2.0 \AA$ resolution from a different crystal (space group C2) soaked with AMP (2B8J; see Materials and Methods). As in the previous case, the AMP hydrolysis products are bound to the enzyme in the P1 and P2 sites. However, in this structure, the two AphA subunits present in the asymmetric unit show different conformations of the loop 6 one being in the "open" and the other being in the "closed" conformation observed in the previous structure. This difference appears to be correlated to the presence of the phosphate group in the active site cavity. Indeed, in the open subunit, only the adenosine product is found, while in the other only a phosphate ion is found together with extra electron density confidently interpreted as a coordination compound between a spermine molecule used in the crystallization solution and a $\mathrm{Au}(\mathrm{III})$ ion used in the attempt of obtaining an inactive enzyme by substitution of the native $\mathrm{Mg}$ (II) with gold (see Materials and Methods). The $\mathrm{Au}(\mathrm{III})$-spermine complex is bound into the P2 ring binding cleft (Figure 5). Furthermore, the phosphate ion in the closed subunit shows a new binding mode, since it is not coordinated directly to the $\mathrm{Mg}$ (II) ion. The $\mathrm{Mg}(\mathrm{II})$ ion displays the "native" octahedral coordination by being bound to Asp44, Asp46 and Asp167 and to three water molecules. The interaction between the phosphate and $\mathrm{Mg}$ (II) is now mediated by two metal-coordinated water molecules that bridge the two species, as shown in Figure 6. This
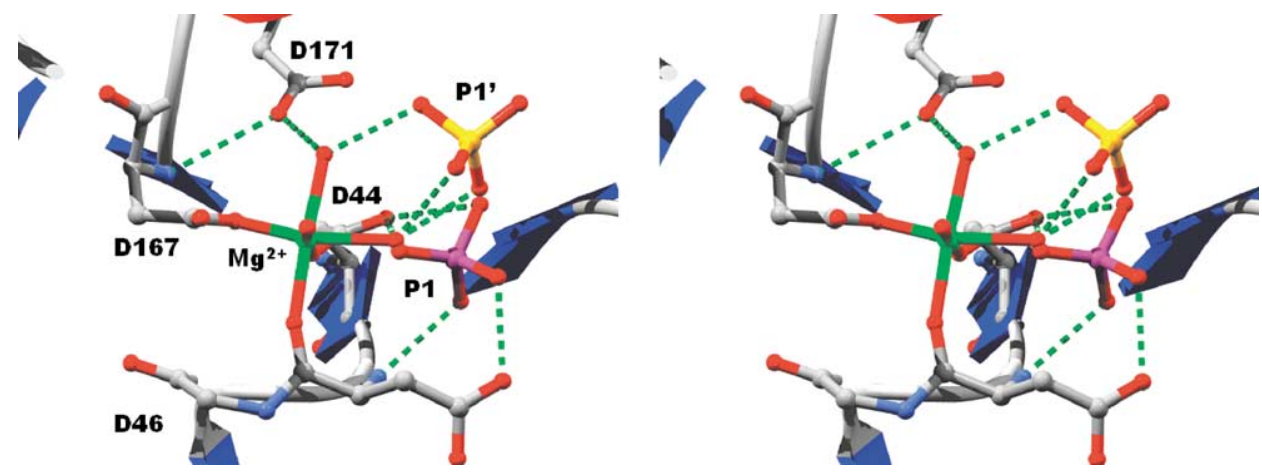

Figure 6. Comparison by least-squares fitting of the structures of AphA-ADN- $P_{i}$ ternary complex with the AphA- $P_{i}$ binary complex. The different binding of the phosphate molecules (P1, magenta; $\mathrm{P} 1$ ', yellow) in the two structures are shown. 
new phosphate-binding site will be denoted the P1' site.

In this subunit, Lys152 is involved, as in the previous case, in tight hydrogen bonds with Asp44, Asp171, Val111 and phosphate, confirming that these interactions stabilize the closed conformation of loop 6.

The Asn150 is $\mathrm{H}$ bonded to Asp170 carbonyl oxygen atom and to Thr173 side-chain. Asp145 is H bonded to spermine, which occupies the same site of the adenine molecule in the previous structure.

\section{AphA complexed with dCMP}

Among the known AphA nucleotide substrates, $5^{\prime}$-CMP and 5'-dCMP are those with which the enzyme exhibits the higher apparent $K_{\mathrm{M}}$ values $\left(K_{\mathrm{M}}=138 \mu \mathrm{M}\right.$ and $35 \mu \mathrm{M}$, respectively), which are about 50 times higher than those shown with $5^{\prime}$-AMP and $5^{\prime}$-dAMP. ${ }^{21}$ We have then determined the crystal structure of the complex between AphA and $5^{\prime}-\mathrm{dCMP}$ (PDB code 1RMQ) with the aim to ascertain if the above-mentioned differences in $K_{\mathrm{M}}$ correspond to different binding modes. Once again, the crystal structure shows a ternary complex between the enzyme and the hydrolysis products of 5'-dCMP: deoxycytidine (dCTN) and phosphate. The two independent AphA subunits present in the crystal asymmetric unit (space group C2) differ by the ligands bound into the active site. In one subunit only dCTN is bound to the P2 site, while the other subunit binds both the nucleoside and a phosphate anion in the P2 and P1 sites, respectively, similar to what has been observed in the AphA-adenosinephosphate ternary complex. The former subunit has loop 6 in the open conformation, while the latter has the loop 6 in the closed conformation displaying the described pattern of $\mathrm{H}$ bonds.

The binding of the dCTN found in this structure is shown in Figure 7. The deoxycytidine binding to the P2 site shows small differences with respect to that of adenosine. The smaller dimensions of the pyrimidine ring and the presence of a carbonyl oxygen atom on it, allow the occurrence of two $\mathrm{H}$ bonds with two water molecules revealed in the cavity. These water-mediated $\mathrm{H}$ bonds link the cytidine $\mathrm{O} 2$ atom to Ser52 and Asp47 side-chains. These H-bonds are not present in the adenosinephosphate-AphA ternary complex. The present structure confirms the P2 site as the binding site of the nucleoside product.

Two different qualitative explanations of the high $5^{\prime}$-dCMP $K_{\mathrm{M}}$ can be suggested on the basis of the crystallographic results: (i) the extra binding enthalpy due to the two $\mathrm{H}$ bonds made by dCTN is not compensated by the higher entropic contribution achieved when the two water molecules are freed from the cavity upon binding of $5^{\prime}$-AMP; (ii) the observed extra $\mathrm{H}$ bonds stabilize the binding of the dCTN product, which competes with the substrate $5^{\prime}$-dCMP for the cavity, leading to a higher apparent $K_{\mathrm{M}}$. Furthermore, the smaller pyrimidine ring of cytidine may optimise the stacking interactions with Phe56 and Tyr193 contributing to a lower free energy of binding. This means that the deoxycytidine/ cytidine products might have a higher affinity for the P2 site than the deoxyadenosine/adenosine products, and hence could compete more strongly with substrates for binding, explaining the higher $K_{M}$ values exhibited by $5^{\prime}$-dCMP and $5^{\prime}$-CMP compared with $5^{\prime}$-dAMP and $5^{\prime}$-AMP. In this respect, is worth mentioning that competition measurements of the effect of dCTN on $5^{\prime}$-dCMP hydrolysis show that $\mathrm{dCTN}$ behaves as a competitive inhibitor showing an increase of the substrate $K_{\mathrm{M}}$ and unaltered $V_{\max }$ (unpublished results).

\section{AphA-osmate complex}

One of the possible reaction pathways of phosphoester hydrolysis by a phosphatase involves the formation of a phosphoacyl (phosphonate) intermediate obtained by the nucleophilic attack of an Asp carboxylate to the phosphorus atom of the substrate. ${ }^{8,9}$ In the hope to attain the structure of a reaction intermediate analogue, we have determined the crystal structure of the adduct of AphA with the $\left[\mathrm{OsO}_{4}\right]^{2-}$ anion, which is a phosphate analogue.

The asymmetric unit (space group C2; Table 2) contains two independent AphA subunits that
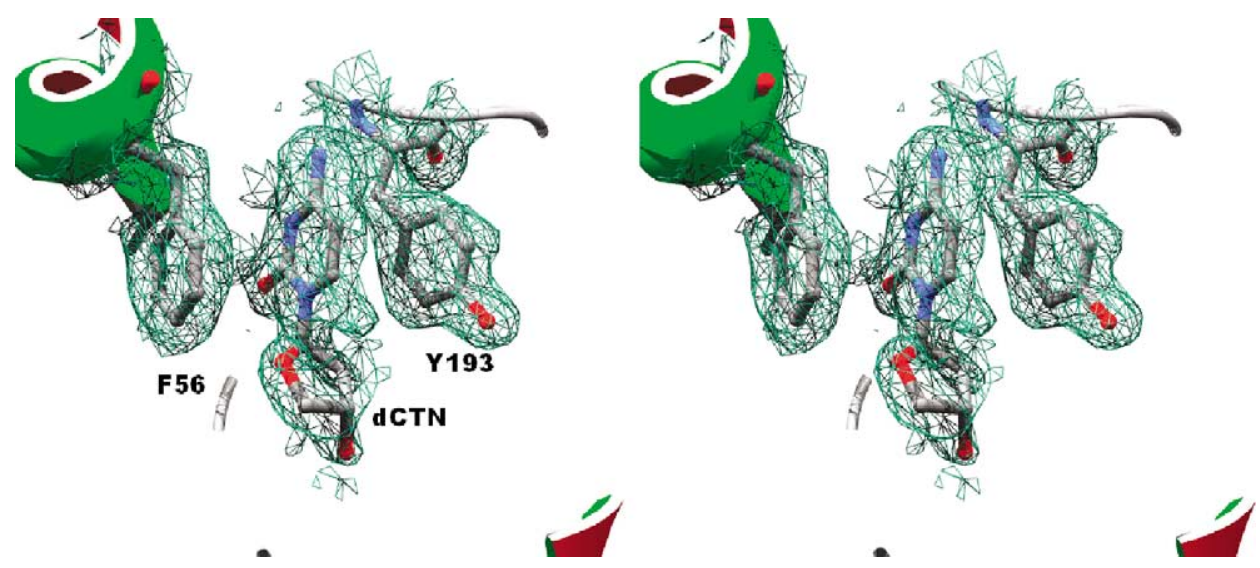

Figure 7. Detail of the $2 F_{\mathrm{o}}-F_{\mathrm{c}}$ electron density map showing the AphA-dCNT complex. The map is contoured at $1.0 \sigma$. 
display the usual structure of the enzyme. However, in both active sites, two new features are present: (i) the native $\mathrm{Mg}$ (II) ion has been replaced by the $\mathrm{Co}$ (II) ion used in the crystallization solution to obtain crystals of better quality (see Materials and Methods); and (ii) a large electron density of approximately tetrahedral shape is found between the Co(II) and Asp44. The replacement of the native $\mathrm{Mg}$ (II) ion by cobalt has been ascertained during the refinement as the temperature factors of the putative magnesium atom moved towards zero. On the contrary, the cobalt temperature factors behave well, converging to values very close to those of the other atoms in the active site. An anomalous Fourier difference map computed with data collected at the Os absorption peak energy $(1.1398 \AA)$ shows a $15 \sigma$ maximum in correspondence of the centre of a tetrahedrally shaped electron density close to the cobalt binding site. This indicates that an Os species is bound to this place (Figure 8). In subunit $\mathrm{A}$, the corresponding electron density can be modelled with a stretched tetrahedral $\mathrm{OsO}_{4}$ moiety with one of the oxygen atoms bridging between cobalt and osmium, and another belonging to the Asp44 side-chain, while in subunit B a square pyramid involving the same ligands and one extra oxygen atom provides a better model. The refinement indicates that the osmate group is bound in both subunits with partial occupancy and a value of 0.5 has been used. Other osmium-binding sites, still with partial occupancy, are located at His22 and His131 of both subunits. The distance between Os and the oxygen atom bridging it to the catalytic metal site occupied by cobalt, is $1.8-2.0 \AA$, which is the usual Os-O single bond distance, while the distances to the other oxygen atoms that complete the Os coordination are far longer, being in the 2.3-2.5 $\AA$ range. The uneven distribution of the distances observed around osmium is most probably due to the static disorder present in the site due to the partial occupancy of the osmate species. Indeed the ligands at shorter distance refine well with partial occupancy of 0.5 , while the others refine at full occupancy. In any case, the relevant aspect of this structure is that it demonstrates the existence of a covalent bond between the osmate analogue of phosphate and the carboxylate oxygen atom of Asp44.

\section{Discussion}

\section{Hypothesis on the catalytic mechanism and comparison with other phosphatases}

The set of X-ray structures presented here provides sufficient evidence to explain the affinity of AphA for aromatic phosphomonoesters and to outline a proposal for the catalytic mechanism of their hydrolysis. The structural comparison with the other Mg-dependent phosphatases of the HAD superfamily reveals common mechanistic aspects for these enzymes and the sequence similarity allows us to extend the mechanistic proposal to all class B phosphatases.

Three distinct mechanisms are possible in phosphoester hydrolysis and phosphoryl transfer reactions: ${ }^{9}$ (i) a dissociative, $\mathrm{S}_{\mathrm{N}} 1$ type, mechanism; (ii) an associative, $\mathrm{S}_{\mathrm{N}} 2$ type mechanism; and (iii) a concerted mechanism. The first two cases imply the formation of a stable intermediate, while no intermediate is formed in the concerted mechanism. In the $\mathrm{S}_{\mathrm{N}} 1$ pathway, a direct transfer occurs between the donor and acceptor molecules bound to the enzyme in a ternary complex through a metaphosphate-like transition state (TS), while the $\mathrm{S}_{\mathrm{N}} 2$ pathway requires a two-step reaction, where the enzyme acts as intermediate acceptor of the phosphoryl group by forming a covalent adduct with it and encompasses two five-coordinate phosphorus transition states. ${ }^{22}$ The latter mechanism appears to operate in enzymes where a divalent metal ion is coordinated to the attacked phosphate. $^{23}$

All three mechanisms are possible in enzymecatalysed reactions, ${ }^{9}$ and it appears that the $\mathrm{S}_{\mathrm{N}} 2$
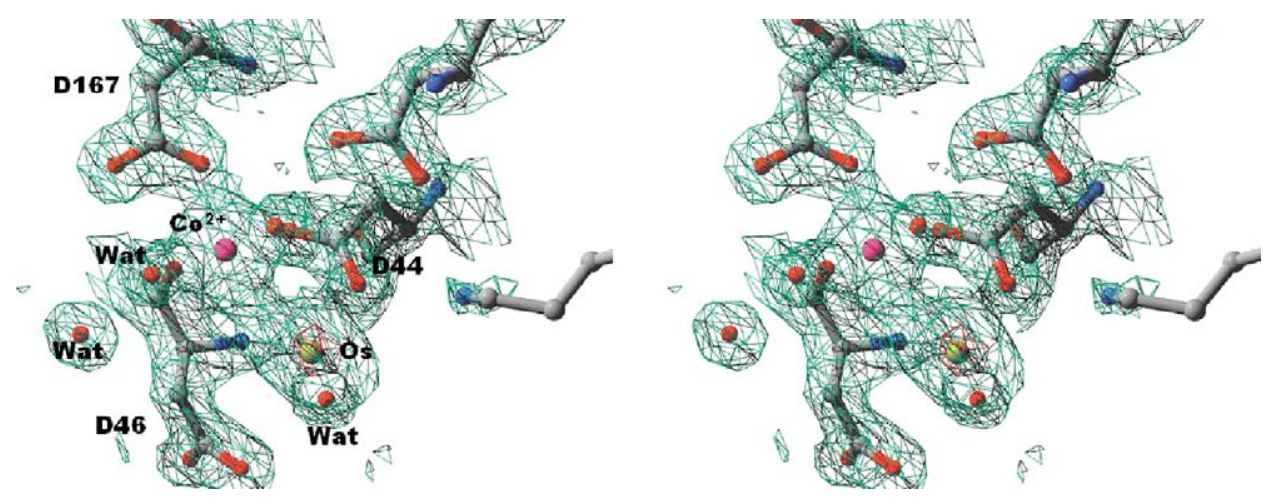

Figure 8. AphA-osmate adduct. The $2 F_{\mathrm{o}}-F_{\mathrm{c}}$ map contoured at $1.0 \sigma$ (cyan), calculated with phases from the final refined model of the complex at $2.0 \AA$ resolution. Superimposed (red) is the anomalous difference Fourier map calculated with coefficients $\Delta$ ano (Os peak energy) and contoured at $15 \sigma$, demonstrating that osmium is bound into the active site. 

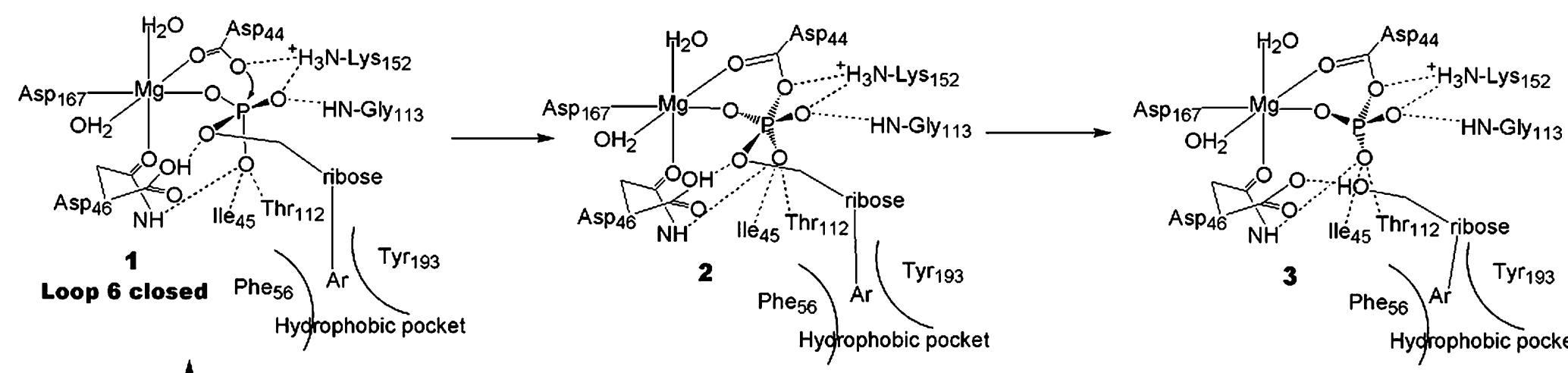

3

Phe 56
Hygrophobic pocket
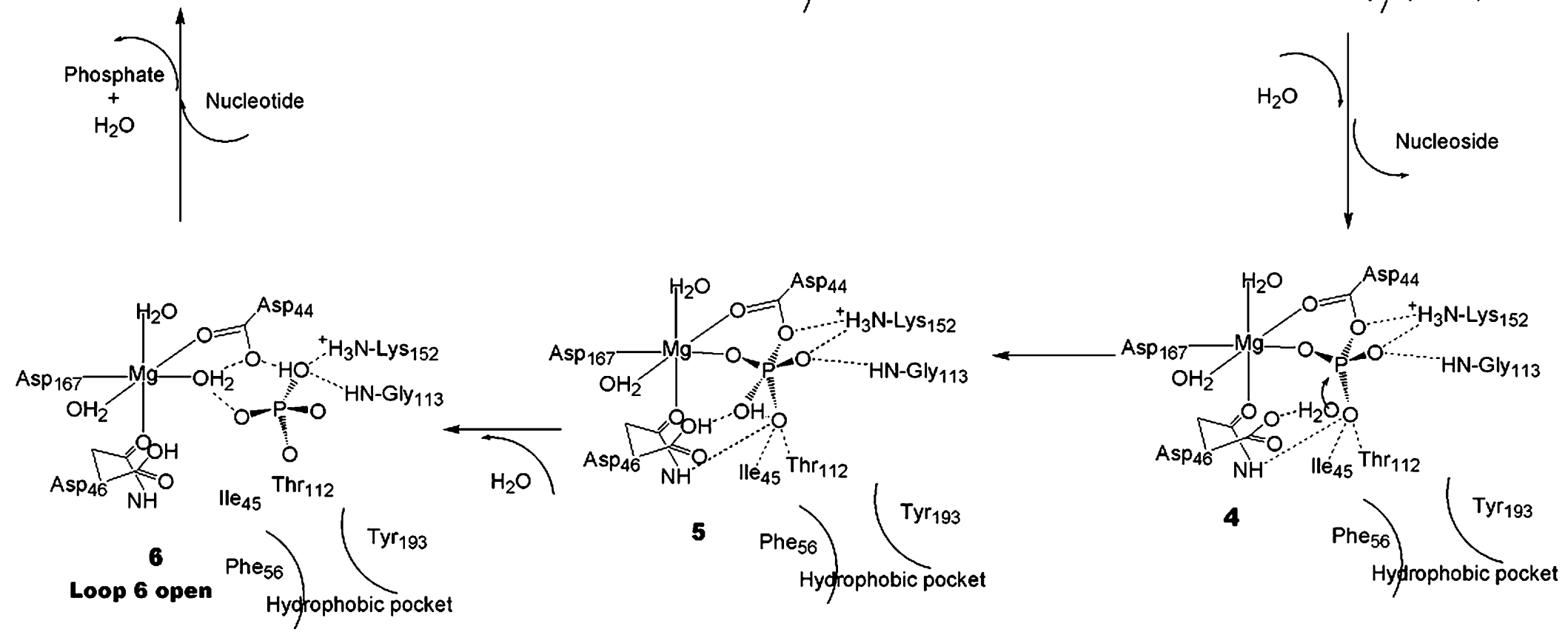

Figure 9. Proposed catalytic pathway for AphA and class B phosphatases. The details of the mechanism concerning the protonation state of the amino acid residues involved in catalysis and of the substrate and reaction products are based on assumptions compatible with the X-ray evidence on hydrogen bonds. 
pathway occurs in the AphA-related enzyme $\beta$-phosphoglucomutase ( $\beta$-PGM), as suggested by the $X$-ray structure of an enzyme-phosphorane intermediate. ${ }^{24}$ However, this view has been disputed; a possible misinterpretation of the $\beta$-PGM structure has been suggested. ${ }^{25}$ Recently, a confirmation of the enzyme-phosphorane intermediate presence in the active site of $\beta$-PGM has been published, eventually settling the controversy. $^{26}$

The structure of the osmate adduct of AphA, where an osmate species is covalently linked to Asp44 bridging it to $\mathrm{Mg}(\mathrm{II})$, constitutes evidence in favour of the formation of a phospho-aspartyl intermediate in the AphA catalytic cycle, where Asp44 acts as a nucleophile towards the substrate activated by the coordination bond to $\mathrm{Mg}^{2+}$. In this hypothesis, the catalytic mechanism of AphA should pass through the formation of the phosphoenzyme and the subsequent hydrolysis of the product phosphate, as suggested by the structural similarity of the AphA catalytic site with that of $\mathrm{PSP}^{5} \mathrm{dNT}^{8}$ and $\beta$-PGM. ${ }^{24}$ Figure 9 reports our proposal for the catalytic mechanism of AphA as suggested by the structural data.

Our results indicate that the catalysis of phosphomonoesters by AphA entails the cyclic movement of loop 6 . The loop 6 open position allows the substrate aromatic moiety to bind into the hydrophobic pocket between Phe56 and Tyr193, and the phosphate group oriented towards the $\mathrm{Mg}$ (II) ion (Figure 9, step1), while the closed position locks the substrate in the active site and facilitates the phosphoryl transfer reaction (Figure 9, steps 2-4). We do not have direct evidence of the existence of the five-coordinate transition state, but the structure at atomic resolution of the AphA-ADN-P $P_{i}$ ternary complex shows the Asp $44 \mathrm{O}^{\delta 1}$ atom at only $2.97 \AA$ from the phosphate phosphorus atom prone to a nucleophilic attack (Figure 9, step 1). In this step, loop 6 is in the closed position with Lys152 H bonded to the nucleophile Asp $44 \mathrm{O}^{\delta 1}$ and to Asp171 $\mathrm{O}^{\delta 1}$ as well as to a phosphate oxygen atom (Figure 10). The role of Lys152, assisted by Asp171, in docking the substrate, in stabilizing the negative charge that develops in the transition state and in stabilizing the closed conformation is clear. On the other hand, the presence of the $\mathrm{H}$ bond between Asp44 $\mathrm{O}^{\delta 1}$ and Lys152 should weaken the carboxylate nucleophilicity and disfavour the reaction. The structure suggests that this disadvantage is overwhelmed by the fact that, by providing a second bridge between Asp44 and the phosphate, Lys152 brings the two reagents in close contact, creating a "concentration" effect similar to what is commonly seen in metal ion-catalysed hydrolytic reactions. In this respect, the $\mathrm{Mg}^{2+}$-Asp44-Lys152 triad can be considered analogous to a catalytic dinuclear metal centre in dimetallic hydrolases, ${ }^{27,28}$ where the metal ions have the double role to bind and activate the substrate and to generate and bind the nucleophile, so that the two species are in close contact. It is noteworthy that the above triad is both sequence and structurally conserved in all magnesium phosphatases-phosphotransferases of the HAD superfamily of known structure. .,5,8,29 $^{-1}$

A second relevant structural motif present in the AphA active site is the cluster composed by the residues Asp46-Arg114-Asp85 shown in Figure 10. All three amino acid residues are conserved in the class B and the double salt-link that connects Arg114 to the carboxylate groups of Asp46 and Asp85 appears to be engineered to maintain the side-chain of Asp46 in a rigid orientation, so as to direct one of the oxygen atom's electron lone pairs towards the P1 site (Figure 10). The structural data indicate for Asp46 a possible role as the general acid/base catalyst needed to transfer a proton to the leaving nucleoside product (Figure 9, steps 2 and 3) and, later, to activate a water molecule towards a nucleophilic attack on the acylphosphate to create the $P_{i}$ product, possibly in cooperation with Thr112 (Figure 9, steps 46). The Asp46Arg114-Asp85 arrangement is not conserved neither in PSP, where, in place of Arg114, a threonine residue (Thr21) ${ }^{5}$ is $\mathrm{H}$ bonded to Asp13 (the structural equivalent of AphA Asp46), or in dNT-2, where a lysine residue (Lys143) takes this place. $^{8}$ In both cases there is no structural equivalent of AphA Asp85. In the mechanism
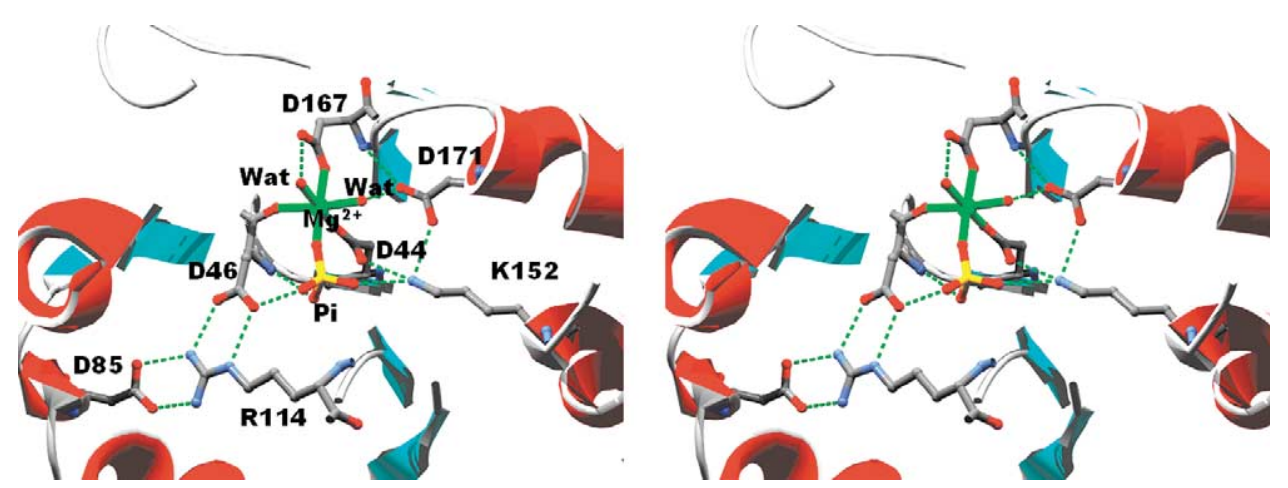

Figure 10. Detail of the structure of the ternary complex AphA-P - ADN showing the phosphate binding and the Asp46-Arg114-Asp85 triad with the relative H bonding scheme (broken green bonds). 
proposed for PSP, ${ }^{5}$ Asp13 moves from its position in the native enzyme to reach the substrate and it is assumed to be protonated in order to provide the hydrogen atom for the leaving serine product. In the dNT-2 mechanism, Asp43 (equivalent to AphA Asp46 and PSP Asp13) is proposed to bind the substrate esteric oxygen atom and to play the same role of PSP Asp13 in protonating the leaving deoxyribose. ${ }^{8}$ In all native and complexed AphA structures, Asp46 occupies the same position, always salt-linked to Arg114, this peculiar arrangement might explain the different $\mathrm{pH}$-dependence of the catalytic activity between AphA, dNT-2 and PSP. While AphA and dNT-2 activity is maximal at acidic $\mathrm{pH}$ (6.5 and 5.0-5.5, respectively), ${ }^{30} \mathrm{PSP}$ has maximum activity at $\mathrm{pH} 7.5 .^{5}$ We propose that the salt-link with Arg114 increases the $\mathrm{pK}$ a of Asp46 so that it can be protonated at slightly acidic $\mathrm{pH}$ and able to play its role as a general acid/base at intermediate $\mathrm{pH}$ with respect to dNT2 and PSP.

Finally, the structure of the binary AphAphosphate complex, showing the phosphate $\mathrm{H}$ bonded to a Mg-coordinated water molecule further away from the Asp44 side-chain, might mimic the leaving phosphate group after the phosphoaspartyl bond hydrolysis. Besides the $\mathrm{H}$ bonds to two of the water molecules from the magnesium coordination sphere (Figure 6), phosphate is bridged by a further water molecule to the side-chains of Asp46 and Thr112, the latter water molecule possibly representing the Asp46/Thr112activated nucleophile responsible for the hydrolysis of the phosphoaspartyl bond. In this structure, the phosphate anion is still $\mathrm{H}$ bonded to the side-chain of Lys152 and appears to be ready to leave the active site upon opening of the loop 6 cover.

\section{Concluding Remarks}

The set of X-ray structures presented allows us to propose a catalytic mechanism for the class B phosphatases of the DDDD superfamily that is similar to that proposed for other $\mathrm{Mg}$ (II)-dependent phosphatases like PSP and dNT-2, and to possibly explain the different $\mathrm{pH}$ activity profile of AphA with respect to that of the former phosphatases.

The structural comparison among the different bacterial and eukaryotic Mg-phosphatases from different families gives evidence for the presence of common motifs such as motifs $1-3$ of the HAD superfamily. However, the AphA structures also identify the differences existing in the substrate binding pocket which discriminate the bacterial class B phosphatases of the DDDD superfamily from the others. In this respect, it should be underlined that AphA and the other class B phosphatases are present only in pathogenic bacterial strains, ${ }^{2,31}$ and may constitute an attractive target for antibiotic design.

\section{Materials and Methods}

\section{Sample preparation and crystallisation}

The AphA gene from E. coli MG1655 was cloned in the E. coli host DH5a for overproduction, as described. ${ }^{32}$ The recombinant AphA protein has been purified following a specifically developed procedure and crystallised as reported. ${ }^{33}$ Diffraction-quality crystals of sizes varying from $0.1 \mathrm{~mm}$ to $0.2 \mathrm{~mm}$ have been obtained from a sittingdrop setup with drops containing $2 \mu \mathrm{l}$ of enzyme solution $\left(10 \mathrm{mg} / \mathrm{ml}\right.$ in $\mathrm{CH}_{3} \mathrm{COONa}$ buffer at $\left.\mathrm{pH} 7.0\right)$ and $2 \mathrm{ml}$ of a precipitant solution $(17-22 \%(\mathrm{w} / \mathrm{v})$ polyethylene glycol (PEG) $6000,1 \mathrm{mM} \mathrm{MgCl}$ ). The drops were equilibrated at room temperature $\left(20^{\circ} \mathrm{C}\right)$ against $100 \mu \mathrm{l}$ of the precipitant solution.

The native AphA crystals obtained with the above protocol are not of constant quality, for this reason we have made use of additives like $0.1 \%(\mathrm{v} / \mathrm{v})$ spermine and $1 \mathrm{mM} \mathrm{CoCl}{ }_{2}$ or detergents like $0.1-0.3 \%(\mathrm{v} / \mathrm{v}) \beta$-octylglucoside to obtain more ordered crystals.

The AphA derivatives were prepared by soaking the native crystals at room temperature in $50 \mathrm{mM}$ $\mathrm{CH}_{3} \mathrm{COONa}$ buffer ( $\mathrm{pH} 7.0$ ), 35\% (w/v) PEG 6000 containing the different substrates (AMP or dCMP) at a concentration of $50 \mathrm{mM}$. One batch of the $\mathrm{Co}$ (II)-treated crystals was soaked with $10 \mathrm{mM} \mathrm{K}_{2} \mathrm{OsO}_{4}$ and the crystal providing the AphA-osmate complex was from this batch. Other native crystals were soaked in $5 \mathrm{mM} \mathrm{AuCl} \mathrm{Au}_{3}$ in the attempt to displace the native $\mathrm{Mg}$ (II) ion and to obtain an inactive enzyme able to bind the intact substrate. The crystals acquired a sharp violet colour and were then used for soaking experiments with AMP. The crystal used for the structure determination of the AphA-Au-spermine complex belonged to this batch.

Before data collection, the crystals were flash-frozen in the crystallisation solution plus $12-15 \%(\mathrm{v} / \mathrm{v})$ ethylene glycol under a cold nitrogen stream.

\section{Data collection and processing}

Four monochromatic experiments were performed on the different derivatised AphA crystals (AMP at atomic resolution, osmate, $\mathrm{AMP}$ at medium resolution and $\mathrm{dCMP}$ ); the first two experiments were performed at ESRF beamlines ID29 and ID14-1 (Grenoble, France). The third experiment was carried out at ELETTRA XRD-1 beamline (Trieste, Italy) while the fourth was performed at EMBL BW7A beamline at DESY (Hamburg, Germany).

Table 3 reports the data collection statistics for the four crystals.

In all cases, the presence of the ligand has most probably lowered the "native" I222 symmetry into C2. For every dataset, the data collection strategy was optimised by using the program BEST. ${ }^{34}$ All the datasets were processed using the program MOSFLM, ${ }^{35}$ and scaled using the program SCALA ${ }^{36}$ with the TAILS and SECONDARY corrections on (the latter restrained with a TIE SURFACE command) to achieve an empirical absorption correction.

\section{Structure determination}

All the five structures were solved using the molecular replacement technique; the model used was that of a subunit of $\mathrm{Au}^{3+}$-derivatized AphA (PDB-ID 1N8N) with all the water molecules and ions omitted. The correct orientation and translation of the molecule within the 
Table 3. Crystallographic data collection and refinement statistics

\begin{tabular}{|c|c|c|c|c|}
\hline Compound & $\begin{array}{l}\text { AphA-AMP high } \\
\text { resolution }\end{array}$ & AphA-osmiate & $\begin{array}{l}\text { AphA-AMP medium } \\
\text { resolution }\end{array}$ & AphA-dCMP \\
\hline PDB code & 2B82 & $1 \mathrm{RMQ}$ & 2B8J & 1RMY \\
\hline Beamline & ESRF ID29 & ESRF BM30A & Elettra XRD1 & EMBL BW7A \\
\hline Space group, Z & $C 2,2$ & $C 2,2$ & $C 2,2$ & $C 2,2$ \\
\hline \multicolumn{5}{|l|}{ Cell dimensions } \\
\hline$a(\AA)$ & 90.936 & 91.732 & 89.966 & 91.882 \\
\hline$b(\AA)$ & 66.812 & 66.447 & 66.544 & 66.393 \\
\hline$c(\AA)$ & 86.054 & 91.523 & 91.544 & 90.018 \\
\hline$\beta$ (deg.) & 116.95 & 121.30 & 124.02 & 119.66 \\
\hline Resolution $(\AA)$ & $76.70-1.25(1.28-1.25)$ & $30.58-2.00(2.11-2.00)$ & $49.60-2.03(2.14-2.03)$ & $30.58-1.75$ (1.84-1.75) \\
\hline Total reflections & $292,050(9832)$ & $106,964(14,911)$ & $83,941(9516)$ & $126,331(22,246)$ \\
\hline Unique reflections & $112,327(4912)$ & $31,710(4810)$ & $28,127(3767)$ & $46,824(8319)$ \\
\hline Completeness (\%) & $88.6(58.2)$ & $99.3(97.6)$ & $97.4(89.6)$ & $98.7(98.0)$ \\
\hline$R_{\text {sym }}(\%)$ & $6.9(22.0)$ & $8.9(40.2)$ & $7.7(14.7)$ & $7.5(32.8)$ \\
\hline Multiplicity & $2.6(2.0)$ & $3.4(3.1)$ & $3.0(2.5)$ & $2.7(2.7)$ \\
\hline$I / \sigma(I)$ & $5.1(3.0)$ & $6.2(1.6)$ & $6.2(4.2)$ & $8.5(2.2)$ \\
\hline Resolution range $(\AA)$ & $76.70-1.25(1.28-1.25)$ & $30.00-2.00(2.05-2.00)$ & $49.39-2.03$ (2.09-2.03) & $30.58-1.75(1.79-1.75)$ \\
\hline$R_{\text {cryst }}(\%)$ & $16.5(21.5)$ & $18.7(23.9)$ & $17.1(19.0)$ & $15.6(20.7)$ \\
\hline No. ref. in $R_{\text {freeset }}, R_{\text {free }}(\%)$ & $8072,18.8(23.9)$ & $2265,23.0(29.7)$ & $2330,22.2(28.5)$ & $3918,19.4(24.0)$ \\
\hline No. protein atoms & 3390 & 3283 & 3314 & 3287 \\
\hline No. metal ions & 2 & 6 & 4 & 2 \\
\hline No. ligand atoms & 67 & - & 38 & 37 \\
\hline No. water molecules & 855 & 297 & 209 & 781 \\
\hline Mean $B$-factor $\left(\AA^{2}\right)$ & 13.6 & 26.18 & 18.58 & 14.50 \\
\hline \multicolumn{5}{|l|}{ RMSD from ideal } \\
\hline Bond lengths $(\AA)$ & 0.006 & 0.026 & 0.017 & 0.017 \\
\hline Bond angles (deg.) & 1.39 & 1.94 & 1.59 & 1.50 \\
\hline Dihedrals (deg.) & 5.17 & 8.20 & 6.16 & 5.50 \\
\hline Chiral volumes $\left(\AA^{3}\right)$ & 0.077 & 0.148 & 0.108 & 0.112 \\
\hline Planes $(\AA)$ & 0.004 & 0.010 & 0.010 & 0.007 \\
\hline Est. error on coord. $(\AA)$ & 0.04 & 0.18 & 0.17 & 0.11 \\
\hline
\end{tabular}

Values in parentheses are for the highest resolution shell.

crystallographic unit cell was determined with standard Patterson search techniques, ${ }^{37,38}$ as implemented in the program MOLREP. ${ }^{39,40}$ The program provided an evident solution for the positioning for the two molecules in the asymmetric unit with a correlation factor of 0.6 . The first two or three $\mathrm{N}$-terminal residues (depending on the dataset) present in the construct were not visible in the electron density maps and are not part of our models; in all cases, the C-terminal residue had a very clear electron density.

The refinement was carried out by using REFMAC5. ${ }^{41,42}$ Non-crystallographic symmetry (NCS) restraints were always used in the very first stages of the refinement. At an $R$-factor of about 0.32 the NCS symmetry was released for the final refinement cycles. Between the refinement cycles, the model was subjected to manual rebuilding using XtalView. ${ }^{43}$ The same program has been used to model the various ligands. Water molecules have been added in all cases by using the standard procedures within the ARP/wARP suite. ${ }^{44,45}$

The stereochemical quality of the refined models was assessed using the program PROCHECK. ${ }^{46}$ The Ramachandran plots are very good in all cases, with no residue in a disallowed region. The stereochemistry of the refined models is thus within the standard tolerance, with RMSD values ranging from $0.006-0.026 \AA$ for bond lengths and from 1.39-1.94 for bond angles.

Table 3 reports all the refinement statistics.

\section{Protein Data Bank accession codes}

The coordinates and the structure factors for the four complexes have been deposited in the Protein Data Bank under the accession codes 1RMQ, 2B82, 1RMY and 2B8J.

\section{Acknowledgements}

We acknowledge funding from the EU (for support of the work at EMBL Hamburg through the HPRI-CT-1999-00017 grant), and from the Italian MIUR (PRIN 2003 to S.M., V.C., M.B. and C.F.). We acknowledge the support and assistance from ESRF-Grenoble and Elettra-Trieste for the data collection.

\section{References}

1. Thaller, M. C., Schippa, S. \& Rossolini, G. M. (1998). Conserved sequence motifs among bacterial, eukaryotic, and archaeal phosphatases that define a new phosphohydrolase superfamily. Protein Sci. 7, 1647-1652.

2. Calderone, V., Forleo, C., Benvenuti, M., Thaller, M. C, Rossolini, G. M \& Mangani, S. (2004). The first structure of a bacterial class B acid phosphatase reveals further structural heterogeneity among phosphatases of the haloacid dehalogenase fold. J. Mol. Biol. 335, 761-773.

3. Wang, W., Kim, R., Jancarik, J., Yokota, H. \& Kim, S. H. (2001). Crystal structure of phosphoserine phosphatase from Methanococcus jannaschii, a hyperthermophile, at $1.8 \AA$ resolution. Structure, 9, 65-71.

4. Wang, W., Cho, H. S., Kim, R., Jancarik, J., Yokota, H., Nguyen, H. H. et al. (2002). Structural characterization of the reaction pathway in phosphoserine phosphatase: crystallographic "snapshots" of intermediate states. J. Mol. Biol. 319, 421-431. 
5. Kim, H. Y., Heo, Y. S., Kim, J. H., Park, M. H., Moon, J., Kim, E. et al. (2002). Molecular basis for the local conformational rearrangement of human phosphoserine phosphatase. J. Biol. Chem. 277, 46651-46658.

6. Shin, D. H., Roberts, A., Jancarik, J., Yokota, H., Kim, R., Wemmer, D. E. \& Kim, S. H. (2003). Crystal structure of a phosphatase with a unique substrate binding domain from Thermotoga maritima. Protein Sci. 12, 1464-1472.

7. Kim, Y., Yakunin, A. F., Kuznetsova, E., Xu, X., Pennycooke, M., Gu, J. et al. (2004). Structure- and function-based characterization of a new phosphoglycolate phosphatase from Thermoplasma acidophilum. J. Biol. Chem. 279, 517-526.

8. Rinaldo-Matthis, A., Rampazzo, C., Reichard, P., Bianchi, V. \& Nordlund, P. (2002). Crystal structure of a human mitochondrial deoxyribonucleotidase. Nature Struct. Biol. 9, 779-787.

9. Allen, K. N. \& Dunaway-Mariano, D. (2004). Phosphoryl group transfer: evolution of a catalytic scaffold. Trends Biochem. Sci. 29, 495-503.

10. Collet, J. F., Stroobant, V., Pirard, M., Delpierre, G. \& van Schaftingen, E. (1998). A new class of phosphotransferases phosphorylated on an aspartate residue in an amino-terminal DXDX(T/V) motif. J. Biol. Chem. 273, 14107-14112.

11. Koonin, E. V. \& Tatusov, R. L. (1994). Computer analysis of bacterial haloacid dehalogenases defines a large superfamily of hydrolases with diverse specificity. Application of an iterative approach to database search. J. Mol. Biol. 244, 125-132.

12. Baker, A. S., Ciocci, M. J., Metcalf, W. W., Kim, J., Babbitt, P. C., Wanner, B. L. et al. (1998). Insights into the mechanism of catalysis by the $\mathrm{P}-\mathrm{C}$ bond-cleaving enzyme phosphonoacetaldehyde hydrolase derived from gene sequence analysis and mutagenesis. Biochemistry, 37, 9305-9315.

13. Morais, M. C., Zhang, W., Baker, A. S., Zhang, G., Dunaway-Mariano, D. \& Allen, K. N. (2000). The crystal structure of Bacillus cereus phosphonoacetaldehyde hydrolase: insight into catalysis of phosphorus bond cleavage and catalytic diversification within the HAD enzyme superfamily. Biochemistry, 39, 10385-10396.

14. Lu, Z., Dunaway-Mariano, D. \& Allen, K. N. (2005). HAD superfamily phosphotransferase substrate diversification: structure and function analysis of HAD subclass IIB sugar phosphatase BT4131. Biochemistry, 44, 8684-8696.

15. Selengut, J. D. \& Levine, R. L. (2000). MDP-1: a novel eukaryotic magnesium-dependent phosphatase. Biochemistry, 39, 8315-8324.

16. Selengut, J. D. (2001). MDP-1 is a new and distinct member of the haloacid dehalogenase family of aspartate-dependent phosphohydrolases. Biochemistry, 40, 12704-12711.

17. Parsons, J. F., Lim, K., Tempczyk, A., Krajewski, W., Eisenstein, E. \& Herzberg, O. (2002). From structure to function: YrbI from Haemophilus influenzae (HI1679) is a phosphatase. Proteins: Struct. Funct. Genet. 46, 393-404.

18. Thaller, M. C., Schippa, S., Bonci, A., Cresti, S. \& Rossolini, G. M. (1997). Identification of the gene (aphA) encoding the class B acid phosphatase/ phosphotransferase of Escherichia coli MG1655 and characterization of its product. FEMS Microbiol. Letters, 146, 191-198.
19. Combet, C., Blanchet, C., Geourjon, C. \& Deleage, G. (2000). NPS@: network protein sequence analysis. Trends Biochem. Sci. 25, 147-150.

20. Aravind, L., Galperin, M. Y. \& Koonin, E. V. (1998). The catalytic domain of the P-type ATPase has the haloacid dehalogenase fold. Trends Biochem. Sci. 23, 127-129.

21. Passariello, C., Forleo, C., Micheli, V., Schippa, S., Leone, R., Mangani, S. et al. (2005). Biochemical characterization of the class $\mathrm{B}$ acid phosphatase (AphA) of Escherichia coli MG1655.. In the press BBA Proteins Proteom.

22. Knowles, J. R. (1980). Enzyme-catalyzed phosphoryl transfer reactions. Annu. Rev. Biochem. 49, 877-919.

23. Yan, H. \& Tsai, M. D. (1999). Nucleoside monophosphate kinases: structure, mechanism, and substrate specificity. Advan. Enzymol. Relat. Areas Mol. Biol. 73, 103-134.

24. Lahiri, S. D., Zhang, G., Dunaway-Mariano, D. \& Allen, K. N. (2003). The pentacovalent phosphorus intermediate of a phosphoryl transfer reaction. Science, 299, 2067-2071.

25. Blackburn, G. M., Williams, N. H., Gamblin, S. J. \& Smerdon, S. J. (2003). Comment on the pentacovalent phosphorous intermediate of a phosphoryl transfer reaction. Science, 301, 1184c.

26. Tremblay, L. W., Zhang, G., Dai, J., DunawayMariano, D. \& Allen, K. N. (2005). Chemical confirmation of a pentavalent phosphorane in complex with beta-phosphoglucomutase. J. Am. Chem. Soc. 127, 5298-5299.

27. Kimura, E. (2000). Dimetallic hydrolases and their models. Curr. Opin. Chem. Biol. 4, 207-213.

28. Ciurli, S., Benini, S., Rypniewski, W. R., Wilson, K. S., Miletti, S. \& Mangani, S. (1999). Structure-function relationships in urease. Coord. Chem. Rev. 190, 331-355.

29. Morais, M. C., Zhang, W., Baker, A. S., Zhang, G., Dunaway-Mariano, D. \& Allen, K. N. (2000). The crystal structure of Bacillus cereus phosphonoacetaldehyde hydrolase: insight into catalysis of phosphorus bond cleavage and catalytic diversification within the HAD enzyme superfamily. Biochemistry, 39, 10385-10396.

30. Gallinaro, L., Crovatto, K., Rampazzo, C., Pontarin, G., Ferraro, P., Milanesi, E. et al. (2002). Human mitochondrial 5'-deoxyribonucleotidase. Overproduction in cultured cells and functional aspects. J. Biol. Chem. 277, 35080-35087.

31. Rossolini, G. M., Schippa, S., Riccio, M. L., Berlutti, F., Macaskie, L. E. \& Thaller, M. C. (1998). Bacterial nonspecific acid phosphohydrolases: physiology, evolution and use as tools in microbial biotechnology. Cell Mol. Life Sci. 54, 833-850.

32. Thaller, M. C., Lombardi, G., Berlutti, F., Schippa, S. \& Rossolini, G. M. (1995). Cloning and characterization of the NapA acid phosphatase/phosphotransferase of Morganella morganii: identification of a new family of bacterial acid-phosphatase-encoding genes. Microbiology, 141, 2-54.

33. Forleo, C., Benvenuti, M., Schippa, S., Thaller, M. C., Rossolini, G. M. \& Mangani, S. (2003). Expression, purification, crystallization and preliminary X-ray characterization of the class B acid phosphatase (AphA) from Escherichia coli. Acta Crystallog. sect. D, 59, 1058-1060. 
34. Popov, A. N. \& Bourenkov, G. P. (2003). Choice of data-collection parameters based on statistic modelling. Acta Crystallog. sect. D, 59, 1145-1153.

35. Leslie, A. G. W. (1991). In Molecular Data Processing (Moras, D., Podjarny, A. D. \& Thierry, J. P., eds), vol. 5, pp. 50-61, Oxford University Press, Oxford.

36. Evans, P. R. (1997). SCALA, continuous scaling program. Joint CCP4 ESF-EACBM Newsletter, 33, 22-24.

37. Rossmann, M. G. \& Blow, D. M. (2000). The detection of sub-units within the crystallographic asymmetric unit. Acta Crystallog. 15, 24-31.

38. Crowther, R. A. (1972). In The Molecular Replacement Method (Rossmann, M. G., ed.), Gordon and Breach, New York.

39. Vagin, A. \& Teplyakov, A. (1997). MOLREP: an automated program for molecular replacement. J. Appl. Crystallog. 30, 1022-1025.

40. Vagin, A. \& Teplyakov, A. (2000). An approach to multi-copy search in molecular replacement. Acta Crystallog. sect. D, 56, 1622-1624.
41. Collaborative Computational Project Number 4 (1994). The CCP4 suite: programs for protein crystallography. Acta Crystallog. sect. D, 50, 760-763.

42. Murshudov, G. N., Vagin, A. A. \& Dodson, E. J. (1997). Refinement of macromolecular structures by the maximum-likelihood method. Acta Crystallog. sect. D, 53, 240-255.

43. McRee, D. E. (1999). XtalView/Xfit-a versatile program for manipulating atomic coordinates and electron density. J. Struct. Biol. 125, 156-165.

44. Lamzin, V. S. \& Wilson, K. S. (1993). Automated refinement of protein models. Acta Crystallog. sect. D, 49, 129-147.

45. Perrakis, A., Morris, R. \& Lamzin, V. S. (1999). Automated protein model building combined with iterative structure refinement. Nature Struct. Biol. 6, 458-463.

46. Laskowski, R. A., MacArthur, M. W., Moss, D. S. \& Thornton, J. M. (1993). PROCHECK: a program to check the stereochemical quality of protein structures. J. Appl. Crystallog. 26, 283-291.

Edited by M. Guss

(Received 30 June 2005; received in revised form 19 October 2005; accepted 25 October 2005) Available online 10 November 2005 